\title{
Identificação de Danos Estruturais Utilizando a Matriz de Flexibilidade Estrutural e Modelo de Superfície de Resposta
}

\author{
Isabela Cristina da Silveira e Silva Rangel ${ }^{1}$ \\ Leonardo Tavares Stutz ${ }^{2}$ \\ Luciano dos Santos Rangel ${ }^{3}$ \\ Programa de Pós-Graduação em Modelagem Computaional, Instituto Politécnico do Estado do \\ Rio de Janeiro, UERJ, Nova Friburgo, RJ \\ Rosilene Abreu Portella Corrêa ${ }^{4}$ \\ Programa de Pós-Graduação em Modelagem Computacional em Ciência e Tecnologia, Instituto do \\ Noroeste Fluminense de Educação Superior, UFF, Santo Antônio de Pádua, RJ
}

\begin{abstract}
Resumo. O presente trabalho aborda um problema de identificação de danos estruturais baseado na análise modal, mais precisamente, na matriz de flexibilidade, em uma viga de Euler-Bernoulli simplesmente apoiada. O Modelo de Superfície de Resposta (MSR) é utilizado para a obtenção da matriz de flexibilidade em substituição ao Modelo de Elementos Finitos (MEF) na formulação do problema inverso da identificação de danos e, para tal, é utilizado o método Evolução Diferencial (ED). Frente aos resultados numéricos obtidos, a estratégia adotada mostrou-se capaz de identificar os danos com elevada acurácia, mostrando assim, sua potencialidade.
\end{abstract}

Palavras-chave. Identificação de Danos Estruturais, Matriz de Flexibilidade, Modelo de Superfície de Resposta.

\section{Introdução}

O contínuo monitoramento da estrutura e a identificação de danos num estágio inicial contribuem para a redução dos custos de manutenção e de reparo, além de aumentar sua confiabilidade e sua vida útil, visto que, a presença de danos compromete o desempenho e a integridade estrutural, podendo colocar vidas humanas em risco e resultam em perdas econômicas consideráveis.

Sendo assim, estudos e ensaios devem ser realizados com intuito de fornecer recursos para uma correta avaliação da integridade da estrutura. Métodos de identificação de danos estruturais e monitoramento de estruturas têm despertado bastante atenção da comunidade científica, esses métodos são classificados em quatro categorias: método de

\footnotetext{
${ }^{1}$ isabelacssilva@yahoo.com.br

${ }^{2}$ ltstutz@iprj.uerj.br

${ }^{3}$ lucianosrangel@yahoo.com.br

${ }^{4}$ rosileneportella@id.uff.br
} 
identificação de danos no domínio do tempo [5], no domínio da frequência [4], métodos baseados na impedância [3] e na análise modal, base para o presente trabalho.

Técnicas de identificação de danos estruturais e monitoramento de estruturas fundamentadas no ajuste de um Modelo de Elementos Finitos (MEF) são constantes na literatura. No entanto, a obtenção de um problema geralmente mal posto e o elevado custo computacional, inerente a essas técnicas, limitam a sua aplicabilidade em estruturas que demandam um modelo de ordem elevada. Para contornar essas dificuldades, na formulação do problema de identificação de danos, pode-se utilizar o Modelo de Superfície de Resposta (MSR) em substituição a um MEF da estrutura.

No presente trabalho, o processo de identificação de danos estruturais considera o ajuste de um MSR de uma viga de Euler-Bernoulli simplesmente apoiada, considerando a matriz de flexibilidade estrutural na formulação do problema inverso. No trabalho são descritos: o modelo matemático para o problema direto de determinação dos parâmetros modais da estrutura, a abordagem do parâmetro de coesão $\beta$ e a base teórica no MSR. E, por fim, são apresentados os resultados numéricos.

\section{Modelagem do Dano e Matriz de Flexibilidade}

Neste trabalho, descreve-se o dano ao longo da viga, por um modelo de dano contínuo onde o campo de danos da estrutura é descrito por meio de um parâmetro nodal de coesão $\beta$ [7]. Esse parâmetro relaciona-se com a ligação entre os pontos materiais e pode ser interpretado como uma medida do estado de coesão local do material, onde $0 \leq \beta \leq 1$. Se $\beta=1$, considera-se que todas as ligações entre os pontos materiais foram preservadas, ou seja, não há defeito na estrutura. Se $\beta=0$, considera-se uma ruptura local, pois todas as ligações entre os pontos materiais foram desfeitas.

A presença de dano estrutural pode resultar em alterações nas propriedades elásticas da estrutura, ou seja, alteração no módulo de elasticidade do material, no momento de inércia de área da seção transversal ou em ambos. Deste modo, o parâmetro de coesão reflete qualquer alteração na rigidez à flexão $E(x) I(x)$ devido a presença de danos

$$
E(x) I(x)=\beta^{e}(x) E_{0} I_{0},
$$

onde: $E_{0}$ e $I_{0}$ são respectivamente, os valores nominais do módulo de elasticidade e do momento de inércia da área da seção transversal. Portanto, matriz de rigidez do MEF da estrutura pode ser escrita como

$$
\mathbf{K}\left(\beta_{h}\right)=\int_{\Omega} \beta(x) E_{0} I_{0} \mathbf{H}^{T}(x) \mathbf{H}(x) d \Omega,
$$

sendo $\mathbf{H}$ o operador diferencial discretizado, $E_{0}$ e $I_{0}$ são, respectivamente, os valores nominais do módulo de elasticidade e do momento de inércia de área, $T$ representa a transposição de uma matriz e $\beta$ o campo de coesão no domínio elástico $\Omega$ da estrutura.

Considerando, por simplicidade, a uniformidade do módulo de elasticidade ao longo da viga, o dano altera apenas as propriedades geométricas da mesma. Sendo assim, o campo 
de coesão pode ser escrito como

$$
\beta(x)=\left(h(x) / h_{0}\right)^{3},
$$

onde: $h_{0}$ e $h(x)$, indicam, respectivamente, a espessura nominal e a espessura da viga na posição $x$.

O vetor de parâmetros nodais de coesão, sendo $n p$ o número total de parâmetros de coesão do modelo, é definido como

$$
\boldsymbol{\beta}=\left[\beta_{1}, \beta_{2}, \ldots, \beta_{n p}\right]^{T} .
$$

A matriz de flexibilidade $\mathbf{G}$ de uma estrutura com $n$ Graus de Liberdade (GDL) é a matriz $n \times n$ definida como a inversa de sua matriz de rigidez $\mathbf{K}$. Portanto, em um problema estático, a matriz de flexibilidade relaciona a força $\mathbf{f}$ aplicada na estrutura com o deslocamento $\mathbf{u}$ resultante,

$$
\mathbf{u}=\mathbf{K}^{-1} \mathbf{f}=\mathbf{G f} .
$$

Embora a matriz de flexibilidade relacione um carregamento estático com o deslocamento resultante, esta pode ser obtida a partir dos parâmetros modais da estrutura como se segue. As frequências naturais e as formas modais não-amortecidas de uma estrutura com $n$ GDL podem ser obtidas a partir do problema de autovalor-autovetor

$$
\mathbf{K} \Phi=\mathbf{M} \Phi \Lambda,
$$

sendo $\mathbf{M}$ a matriz de massa, $\boldsymbol{\Phi}$ a matriz modal da estrutura cuja i-ésima coluna corresponde à forma modal $\phi_{i}$ (i-ésimo autovetor) e $\boldsymbol{\Lambda}$ uma matriz diagonal cujo i-ésimo componente (autovalor) é dado pelo valor quadrático da correspondente frequência natural não-amortecida do sistema, ou seja, $\lambda_{i i}=\omega_{i}^{2}$, todas com dimensão $n \times n$.

Considerando as formas modais da estrutura normalizadas em relação à matriz de massa, onde $\mathbf{I}$ é a matriz identidade, tem-se

$$
\begin{aligned}
\boldsymbol{\Phi}^{T} \mathbf{M} \boldsymbol{\Phi} & =\mathbf{I}, \\
\boldsymbol{\Phi}^{T} \mathbf{K} \boldsymbol{\Phi} & =\boldsymbol{\Lambda} .
\end{aligned}
$$

Partindo da equação (8) e do fato de a matriz de flexibilidade de uma estrutura ser definida como a inversa da matriz de rigidez, tem-se

$$
\mathbf{G}=\left(\mathbf{\Phi} \boldsymbol{\Lambda}^{-1} \boldsymbol{\Phi}^{T}\right)=\sum_{i=1}^{n} \frac{1}{\omega_{i}{ }^{2}} \boldsymbol{\phi}_{i} \boldsymbol{\phi}_{i}^{T} .
$$

Na prática, devido a limitações experimentais, tem-se a seguinte aproximação para a matriz de flexibilidade experimental $\mathbf{G}_{\exp }$ da estrutura,

$$
\mathbf{G}_{\exp }=\sum_{i=1}^{n_{\exp }} \frac{1}{\omega_{i, \exp }^{2}} \boldsymbol{\phi}_{i, \exp } \boldsymbol{\phi}_{i, \exp }^{T},
$$

onde: $n_{\text {exp }}<n$ é o número de modos obtidos do ensaio experimental, $\omega_{i, \exp }$ e $\phi_{i, \exp }$ são, respectivamente, a $i$-ésima frequência natural não-amortecida e forma modal obtidas de um ensaio modal realizado na estrutura supostamente danificada. 


\section{Modelo de Superfície de Resposta}

No MSR, relações explícitas são definidas entre parâmetros da estrutura e respostas de interesse, que no caso, representam os parâmetros nodais de coesão e a matriz de flexibilidade. Desta forma, para uma dada resposta escalar $y$, tem-se

$$
y=f\left(\beta_{1}, \beta_{2}, \ldots, \beta_{n p}\right)+\varepsilon,
$$

onde: $f\left(\beta_{1}, \beta_{2}, \ldots, \beta_{n p}\right)$ representa a relação entre a resposta e as variáveis independentes e $\varepsilon$ sendo o resíduo. Em geral, os fatores (parâmetros do modelo) são codificados como,

$$
x_{i}=\frac{\beta_{i}-\left(\beta_{\min }+\beta_{\max }\right) / 2}{\left(\beta_{\max }-\beta_{\min }\right) / 2}, \quad i=1,2, \ldots, n p,
$$

tal que $x_{i} \in[-1,1]$. Esta relação pode ser aproximada por polinômios de baixa ordem em algumas regiões relativamente pequenas do espaço definido pelas variáveis independentes, sendo então comumente utilizados modelos de primeira ou segunda ordem [4]. Dessa forma, a equação (11) pode ser reescrita como

$$
\hat{y}=f\left(x_{1}, x_{2}, \ldots, x_{n p}\right)+\varepsilon=\hat{b}_{0}+\sum_{i=1}^{n p} \hat{b}_{i} x_{i}+\sum_{i=1}^{n p} \hat{b}_{i i} x_{i}^{2}+\sum_{i<j}^{n p} \sum_{j=2}^{n p} \hat{b}_{i j} x_{i} x_{j} .
$$

Para cada resposta escalar considerada, tem-se uma superfície de resposta. Considerando o método dos mínimos quadrados, estima-se o vetor de coeficientes $\hat{\mathbf{b}}$ por

$$
\hat{\mathbf{b}}=\left(\mathbf{X}^{T} \mathbf{X}\right)^{-1} \mathbf{X}^{T} \mathbf{y}
$$

sendo $\mathbf{y}$ o vetor contendo a resposta $\hat{y}, \mathbf{X}$ a denominada matriz de projeto, cujos componentes são obtidos dos parâmetros codificados $x_{i}$ e $\hat{\mathbf{b}}$ o vetor contendo os coeficientes da função de resposta.

Dê acordo com a referência [2], o MSR constitui-se de combinações de técnicas de Projeto de Experimentos e existem alguns tipos de projeto como: Projeto Fatorial $\left(2^{n p}\right)$, Projeto Composto Central (PCC) e Projeto Fatorial Fracionado $\left(2^{n p-f}\right)$, sendo este último utilizado no presente trabalho. E, foram geradas superfícies do tipo quadrático com interação entre os parâmetros (QI) [4].

\section{Formulação do Problema Inverso}

O problema de identificação de danos pode ser definido, como um problema inverso de estimação de parâmetros, em sua formulação o MSR substitui o MEF, com intuito de otimizar o processo. O problema inverso visa à minimização, em relação ao parâmetro de coesão $\beta$, de um funcional baseado na diferença entre a matriz de flexibilidade experimental, gerada pelo MEF, e a matriz de flexibilidade prevista pelo MSR da estrutura.

Definindo-se o vetor de parâmetros de coesão $\boldsymbol{\beta}, \mathbf{G}_{\text {exp }}$ e $\overline{\mathbf{G}}(\boldsymbol{\beta})$ a matriz de flexibilidade experimental e prevista, respectivamente, o problema inverso deseja minimizar o funcional

$$
\mathcal{F}(\boldsymbol{\beta})=\frac{1}{2}\left(\frac{\mathbf{G}_{e x p}-\overline{\mathbf{G}}(\boldsymbol{\beta})}{\mathbf{G}_{e x p}}\right)^{T}\left(\frac{\mathbf{G}_{e x p}-\overline{\mathbf{G}}(\boldsymbol{\beta})}{\mathbf{G}_{e x p}}\right) .
$$


No presente trabalho, para a resolução do problema inverso, utilizou-se o algoritmo Evolução Diferencial (ED) [6].

\section{$5 \quad$ Resultados Numéricos}

Neste trabalho, foi considerada uma viga de alumínio simplesmente apoiada cujos parâmetros geométricos e materiais são: $1 \mathrm{~m}$ de comprimento, 0,005 m de espessura, 0,05 m de largura, $2700 \mathrm{~kg} / \mathrm{m}^{3}$ de massa específica, $5,21 \times 10^{-10} \mathrm{~m}^{4}$ e $7,26 \times 10^{10} \mathrm{~Pa}$ de momento de inércia de área e módulo de elasticidade, respectivamente.

A viga foi discretizada pelo MEF em 20 elementos bidimensionais do tipo EulerBernoulli, onde cada elemento possui dois GDL, 40 GDL, e um parâmetro de coesão, logo, a viga possui 21 parâmetros nodais de coesão. Considerou-se, tanto na obtenção dos dados experimentais quando na obtenção dos dados previstos, apenas os modos de vibração contidos na faixa de 0 a $450 \mathrm{~Hz}$ e 10 GDL.

No processo de identificação de danos o MEF é utilizado, com os valores dos parâmetros de coesão prescrito de acordo com os cenários de danos considerados, para gerar a matriz de flexibilidade da estrutura danificada, sendo estes, os dados experimentais. No entanto, na estimação de parâmetros realizada pelo problema inverso é utilizado o MSR, a fim de otimizar o processo, sendo estes os dados previstos. A imposição do dano a viga ocorre através da redução na altura relativa da seção transversal $h(x) / h_{0}$, nos nós, contidos no interior das regiões defeituosas. Objetivando-se simular de forma mais realística os dados experimentais, considerou-se a presença de ruído aditivo, de média nula, vide [4].

A Tabela 1, apresenta os cenários de danos que foram considerados neste trabalho.

Tabela 1: Cenários de danos

\begin{tabular}{|c|c|c|c|}
\hline Caso & Posição $(\mathrm{m})$ & $h(x) / h_{0}$ & Nível de ruído (\%) \\
\hline 1 & 0,476 & 0,8 & 1 \\
\hline 2 & 0,476 & 0,8 & 3 \\
\hline 3 & 0,$047 ; 0,809$ & 0,8 & 1 \\
\hline 4 & 0,$047 ; 0,809$ & 0,8 & 3 \\
\hline
\end{tabular}

Nos Caso 1 e 2 considerou-se uma região danificada, com o dano definido pela espessura relativa de $80 \%$ em $x=0,476 \mathrm{~m}$ e com $1 \%$ e $3 \%$ de ruído, respectivamente. Para os Casos 3 e 4, considera-se duas regiões danificadas, em $x=0,047 \mathrm{~m}$ e $x=0,809 \mathrm{~m}$, com mesma redução, de 20\%, na altura relativa e mesmas intensidades de ruídos dos casos anteriores.

Com intuito de validar os resultados encontrados após o processo de estimação de parâmetros, calcula-se os erros relativos dos seis primeiros modos de vibração, antes e depois da estimação dos parâmetros.

Na Figura 1, são apresentados os resultados da média de dez simulações geradas pelo ED para os Casos 1 e 2, e os seus respectivos erros relativos. Pela Figura 1, visualiza-se que a localização e a identificação de danos foram obtidas com acurácia para os cenários descritos. Observa-se também que, após a atualização das variáveis do modelo em ambos casos, houve uma redução significativa nos erros relativos. 


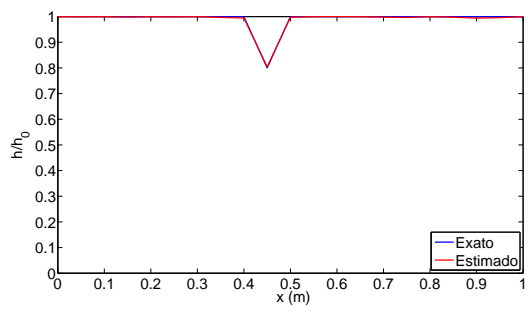

(a) Identificação Caso 1

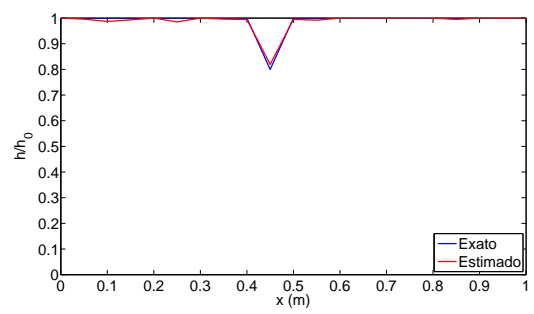

(c) Identificação Caso 2

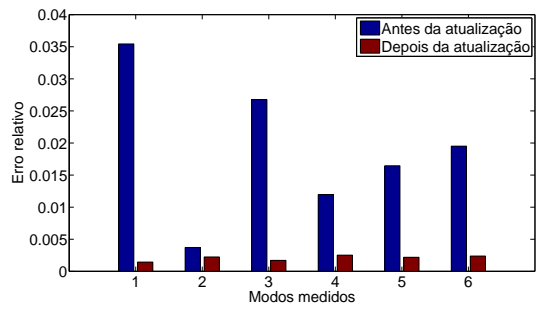

(b) Erro Relativo Caso 1

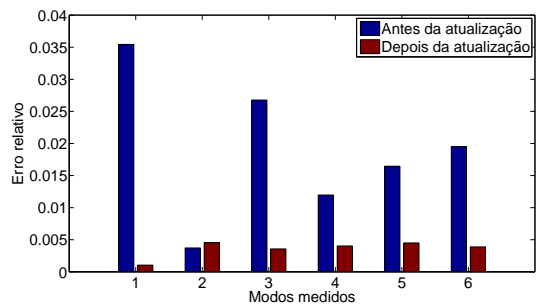

(d) Erro Relativo Caso 2

Figura 1: Identificação de danos para os Casos 1 e 2.

A Figura 2 apresenta a média das dez simulações utilizando o método ED para os Casos 3 e 4, e os seus respectivos erros relativos.

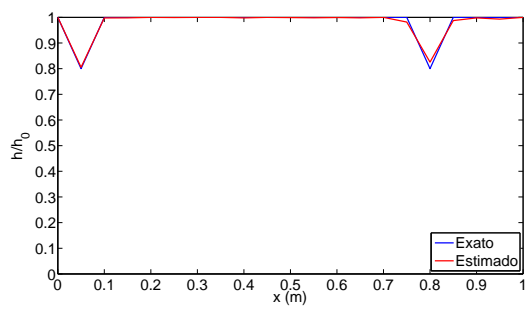

(a) Identificação Caso 3

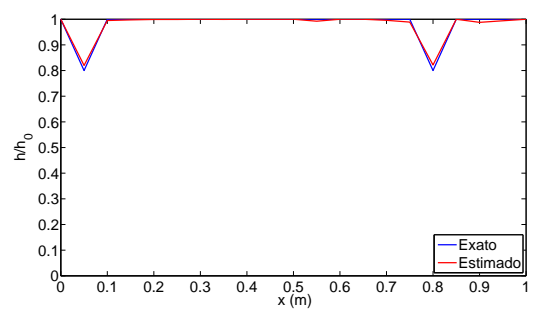

(c) Identificação Caso 4

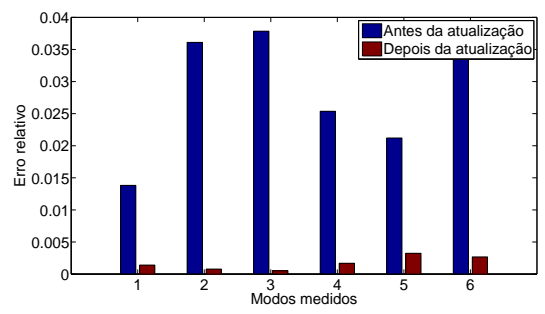

(b) Erro Relativo Caso 3

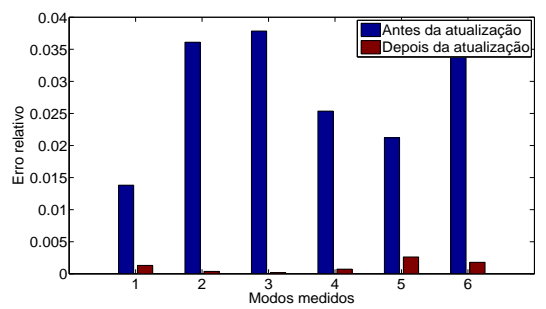

(d) Erro Relativo Caso 4

Figura 2: Identificação de danos para os Casos 3 e 4.

Pelos resultados apresentados na Figura 2, é possível perceber que a estratégia adotada identificou os danos com elevada acurácia e a fim de validar os resultados, observa-se que 
os erros relativos dos modos de vibração considerados, diminuíram consideravelmente após a estimação dos parâmetros nodais de coesão.

\section{Conclusões e Trabalhos Futuros}

Nesse trabalho foi adotado um MSR na identificação de danos estruturais em uma viga de alumínio de Euler-Bernoulli simplesmente apoiada, sendo ajustado para a matriz de flexibilidade estrutural e na estimação de parâmetros do problema inverso foi utilizado o método estocástico ED. Pelos resultados obtidos, observou-se que a estratégia adotada foi capaz de identificar com precisão os cenários de danos em todos os casos, mesmo considerando-se a presença de ruído aditivo nos dados experimentais.

A identificação de danos estruturais utilizando o MSR mostrou-se bastante promissora, portanto, como sugestões para trabalhos futuros, tem-se a aplicação em estruturas do tipo viga com outras condições de contorno e estruturas do tipo placas.

\section{Agradecimentos}

Os autores agradecem às instituições IPRJ/UERJ e INFES/UFF pelo apoio concedido.

\section{Referências}

[1] Q. T. Guo, L. M. Zhang, Finite element model updating based on response surface methodology Proc. 22nd Int. Modal Analysis Conf. (Dearborn, MI), 2004.

[2] P. S. Lopes, Modelagem de problema inverso de detecção de danos por técnicas de identificação de parâmetros e de otimização. Tese de Doutorado em Engenharia Mecânica, Itajubá, pages 135, 2010.

[3] G. Paek, D. J. Inman, Strutural health monitoring using piezoeletric impedance measurements. Philosophical transactions the Royal Society A, 365: 373-392, 2007.

[4] I. C. S. S. Rangel, Identificação de danos estruturais a partir do modelo de superfície de resposta. Dissertação de Mestrado em Modelagem Computacional, Instituto Politécnico, Universidade do Estado do Rio de Janeiro, Nova Friburgo, pages 109, 2014.

[5] L. S. Rangel, I. C. S. S. Rangel, L. T. Stutz, Identificação de danos estruturais utilizando dados no domínio do tempo e critério D-ótimo. Tendências em Matemática Aplicada e Computacional, 16: 209-218, Brasil, 2015.

[6] R. Storn, K. Price, Differential Evolution - A simple and efficient adaptive scheme for global optimization over continuous spaces. Journal of Global Optimization, 11: 341-359, Berkekey, 1997.

[7] L. T. Stutz, D. A. Castello, F. A. Rochinha, A flexibility-based continuum damage identification approach. Journal of Sound and Vibration, 279: 641-667, 2005. 